\title{
Acupuncture inhibits vagal gastric acid secretion stimulated by sham feeding in healthy subjects
}

\author{
G Lux, J Hagel, P Bäcker, G Bäcker, R Vogl, H Ruppin, S Domschke, W Domschke
}

\begin{abstract}
In a prospective randomised study, the effect of acupuncture on sham feeding stimulated gastric acid secretion was investigated. In eight healthy volunteers (five men, three women, mean (SEM) age $26 \cdot 3(4 \cdot 7)$ years) various methods of acupuncture were performed. Apart from the sham procedure, the acupuncture was performed at the classic acupuncture points. Electroacupuncture reduced gastric acid secretion expressed as median (range) significantly during the first 30 minute period to $1.6(0-5 \cdot 2) \mathrm{mmol}$ compared with $3.8(2 \cdot 3-14 \cdot 5) \mathrm{mmol}$ $(p<0.05)$ during control period (sham feeding without acupuncture). Inhibition of gastric acid secretion by electroacupuncture was also significant during the second 30 minute period $(0.2(0-5 \cdot 6) v$ $3.6(0.3-9.1) \mathrm{mmol} ; \mathrm{p}<0.05)$ and for peak acid output $(0.8(0.2-5 \cdot 1) v 7.6(3.4-12.1)$ mmol; $\mathbf{p}<0 \cdot 05)$. Transcutaneous electrical nerve stimulation also resulted in significant reduction of gastric acid secretion during the first 30 minute period $(1 \cdot 0$ (0-3.6) mmol $v$ 3.8 (2.3-14.5) mmol; $\mathbf{p}<0.05)$, and peak acid output $(3.6$ $(1 \cdot 2-12 \cdot 0) v 7 \cdot 6(3 \cdot 4-12 \cdot 1) \mathrm{mmol} ; \mathrm{p}<0 \cdot 05)$. The classic needle acupuncture, laser acupuncture, and sham acupuncture had no significant effect on gastric acid secretion. This study shows firstly that in healthy volunteers, only the versions of acupuncture using more pronounced stimulation (electroacupuncture, transcutaneous electrical nerve stimulation), but not those with only mild stimulation of the nerves (classic needle acupuncture, laser acupuncture), and secondly only acupuncture performed at defined points lead to significant reduction in gastric acid secretion.
\end{abstract}

(Gut 1994; 35: 1026-1029)

Department of

Medicine A,

University of

Erlangen-Nuremberg,

Erlangen, Germany

G Lux

J Hagel

P Bäcker

G Bäcker

$\mathrm{R}$ Vogl

H Ruppin

$S$ Domschke

W Domschke

Correspondence to: Dr G Lux, I Med Clinic, Gotenstraße 1, D-565 Solingen 1, Germany.

Accepted for publication 16 November 1993 in origin. Acupuncture is a Chinese empirical method of healing that can be traced back several thousand years. Insufficient knowledge of the Chinese language, and differences in attitude towards medicine between East and West have led to different evolutions in medicine. In comparison with other methods used by traditional Western medicine, acupuncture seems primitive. This and the fact that the points of stimulation on the skin have no known underlying rational explanation, have combined to prevent a widespread application of the technique in the West. ${ }^{13}$ In particular, however, the mechanism of action and therapeutic successes of acupuncture have only been shown with reproducible, generally accepted scientific techniques in a few studies. $^{2} 4$

Among the gastrointestinal diseases that have been treated with traditional acupuncture are gastric and duodenal ulcer, ${ }^{56}$ motility disorders, ${ }^{7-9}$ and functional complaints affecting the gastrointestinal tract. ${ }^{10}$ There are also reports on accelerated healing of ulcers under acupuncture, ${ }^{1112}$ and on an influence on gastric acid secretion both in humans ${ }^{12}$ and animals. ${ }^{13}$

Gastric acid secretion offers an attractive model for testing the effect of acupuncture because of the ease of reproducible analysis.

In this study, the effect of various forms of acupuncture was studied on gastric acid secretion stimulated by sham feeding in normal human volunteers. In addition to the classic needle acupuncture, other types, such as laser acupuncture, electroacupuncture, and transcutaneous electrical nerve stimulation were used. To exclude a possible unspecific effect resulting from 'suggestion', acupuncture was also performed outside of the acupuncture points described - so called sham acupuncture.

\section{Methods}

Eight healthy subjects (five men, three women, mean (SEM) age $26 \cdot 3(4 \cdot 7)$ years) took part in the study. As one participant failed to complete the study, the data of seven volunteers were considered for evaluation. Different methods of acupuncture used included needle acupuncture, electroacupuncture, laser acupuncture, and transcutaneous electric nerve stimulation. Apart from sham acupuncture the acupuncture was performed at the classic acupuncture points. For the control period sham feeding without acupuncture was given in all subjects in the study.

The subjects gave their written informed consent, and the study protocol was accepted by the ethics commission of the Medical University Hospital of Erlangen.
ANALYSIS OF GASTRIC ACID SECRETION

Gastric acid secretion analysis was started after an overnight fasting period of at least 12 hours. The placing of the gastric tube (outside diameter (OD) $5 \mathrm{~mm}$ ) was verified by a radiological check on the correct position of its tip in the 
lower pole of the stomach. Mechanical aspiration of the gastric acid secretion using an intermittent vacuum of 120 mbar (Hico-Gastrovac 271, Hirtz \& Co, Cologne, Germany) was started after an adaptation phase. The gastric juice was collected in 15 minute periods.

Titration of the gastric juice was performed in $10 \mathrm{ml}$ samples with one tenth normal solution $\mathrm{NaOH}$ until the indicator (phenol red) changed colour at a $\mathrm{pH}$ of $7 \cdot 4$.

\section{CORRECTION FOR LOSS OF VOLUME}

Phenol red was infused continuously into the stomach through a second tube (OD $2 \mathrm{~mm}$ ). The opening of the second tube was located $2 \mathrm{~cm}$ proximal to the most proximal aspiration opening of the gastric tube. Phenol red solution $(40 \mathrm{mg} / 100 \mathrm{ml})$ was instilled at $30 \mathrm{ml} / \mathrm{h}$ by an automatic infusion device (Perfusor V, Braun Melsungen, Germany).

The extinction was determined in the aspirate $(0.5 \mathrm{ml}$ aspirate to which $5.5 \mathrm{ml}$ double distilled water was added), and $1 \mathrm{ml}$ borate buffer $(61.83$ boric acid in $1000 \mathrm{ml}$ double distilled water, adjustment of the $\mathrm{pH}$ to $9 \cdot 2$ with normal solution $\mathrm{NaOH}$ ) at wavelengths of $520 \mathrm{~nm}, 560 \mathrm{~nm}$, and $600 \mathrm{~nm}$.

The total extinction of the aspirated secretion (E-pra) was calculated using the formula:

$\mathrm{E}-\mathrm{pra}=\mathrm{E}-560-\frac{(\mathrm{E}-520+\mathrm{E}-600)}{2}$

The correction factor $F$ is calculated from:

$$
F=\frac{(\mathrm{V} \text {-inst } \times \text { E-pri })}{(\mathrm{V} \text {-asp } \times \text { E-pra })}
$$

Where V-inst=instilled volume; E-pri=extinction of instilled volume; $\mathrm{V}$-asp=aspirated volume; E-pra = extinction of aspirated volume.

By multiplying the titrated acid value by the correction factor, $F$, the loss corrected acid secretion is obtained.

\section{STIMULATION OF GASTRIC ACID SECRETION BY SHAM FEEDING}

Modified sham feeding was carried out using the 'chew and spit' technique. ${ }^{14}$ For stimulation of gastric acid secretion, the test subjects were given white bread with boiled ham to eat; after thorough chewing, the contents of the mouth were spat out and the mouth rinsed with water. Before the study was started, this procedure was used at least five times by each test subject, and the reproducibility of the acid figures obtained was established. During the test period, all subjects had reproducible figures, with a scatter of $<10$ per cent.

\section{ACUPUNCTURE}

Acupuncture was performed in each case by one of the authors (RV), who was an instructor with the German Medical Society for Acupuncture. The acupuncture points were used that in the Chinese acupuncture scheme are stimulated to treat diseases of the stomach $^{13}{ }^{15}$ : bladder 21 (lateral to the lower edge of the spiny process of the 12th thoracic vertebra); stomach 36 (lateral to the edge of the tibia below the knee joint gap); and knee joint 12 (unpaired, in the middle of the line joining the tip of the sternum and the umbilicus).

In each case, the acupuncture was maintained for 30 minutes.

In the case of classic acupuncture and acupuncture with stimulation by electric current, Japanese disposable acupuncture needles (diameter $0.3 \mathrm{~mm}$ ) were inserted into the skin to varying depths (stomach $363-4 \mathrm{~cm}$ deep, bladder $211-3 \mathrm{~cm}$ deep, knee joint 12 to a depth of $1 \mathrm{~cm}$, then $2-3 \mathrm{~cm}$ caudad).

For classic acupuncture, the needles are rotated or wiggled every 10 minutes for 10 seconds. For electrostimulation, the needles were connected to an electrostimulation device (AKU-IMPULS, Pitterling) and stimulated at a period relaxation oscillation $(5 \mathrm{~Hz})$ at a current intensity that produced a 'De-Qi' sensation at the acupuncture point (soreness, numbness or distension) showing correct acupuncture technique. To compensate for adaptation, the intensity was increased every 10 minutes.

For laser acupuncture, a laser acupuncture device (AKUPLAS MFL, MBB, Munich) operating at a frequency of $20 \mathrm{~Hz}$ was used; depth of penetration of the laser beam: $0.8-1.5 \mathrm{~cm}$.

Transcutaneous electrical nerve stimulation was performed with the aid of adhesive electrodes (surface area about $4 \mathrm{~cm}^{2}$ ), and the stimulation device (Tensy Pitterling) with bipolar impulses $(3 \mathrm{~Hz})$, the intensity being increased until a distinctly discernible tingling sensation was produced.

Sham acupuncture was carried out at points for which no acupuncture effect has been described (medial to the insertion of the deltoid muscle, caudal to the iliac crest in the gluteus maximus muscle), and electrical stimulation was applied.

\section{STUDY PROTOCOL}

The test periods for needle acupuncture, electroacupuncture, transcutaneous electrical nerve stimulation, laser acupuncture, sham acupuncture, and the control period were performed in randomised sequence. The test subjects were not informed about the nature of the specific examination.

In each case, the experiments were started at $7.15 \mathrm{am}$, so that the procedures, including acupuncture, always took place in the same period of time. After placement of the gastric tube, the contents of the stomach were aspirated with the patient in a semireclining position, in seven 15 minute periods, that is, over a total period of 105 minutes. The first 15 minute sample was discarded. After another 30 minutes, stimulation of the gastric acid secretion by sham feeding for a 15 minute period was performed, aspiration for the stimulation period started with sham feeding. Stimulated 
acid output included the two 30 minute periods after sham feeding began, peak acid output represented the peak in any 30 minute period from 45-105 minutes ${ }^{16}$. Acupuncture began with insertion of the needles five minutes before the start of the sham feeding, and was performed for a total of 30 minutes.

\section{STATISTICS}

As basal and sham feeding acid output rates show a notoriously skewed distribution Wilcoxon's matched pairs signed rank test was used for comparison. The results are given as medians and their ranges. Calculation was based on 30 minute samples. Statistical significance was assumed at the $5 \%$ value.

\section{Results}

Gastric acid output stimulated by sham feeding was $3.8(2 \cdot 3-14.5) \mathrm{mmol}$ during the first 30 minute period of control period where no acupuncture was performed. This was not statistically different from gastric acid output during sham acupuncture (7.4 (3.1-20.8) $\mathrm{mmol})$, laser acupuncture $(6 \cdot 0(0-9 \cdot 6) \mathrm{mmol})$, and needle acupuncture (8.2 (0.8-28.9) mmol). A significant reduction was seen during electroacupuncture $(1.6(0-5 \cdot 2) \mathrm{mmol})$ $(\mathrm{p}<0.05)$ and during transcutaneous nerve stimulation $(1.0 \quad(0-3.6) \quad \mathrm{mmol}) \quad(\mathrm{p}<0.05)$ (Figure (A)).

During the second 30 minute period acid output was significantly $(\mathrm{p}<0.05)$ inhibited only by electroacupuncture $(0 \cdot 2 \quad(0-5 \cdot 6)$ mmol) compared with the control period ( $3 \cdot 6$ $(0.3-9 \cdot 6) \mathrm{mmol})$. There was no significant influence on gastric acid secretion effected by sham acupuncture $(4 \cdot 8(2 \cdot 2-23 \cdot 5) \mathrm{mmol})$, laser acupuncture $(2 \cdot 2(0-13 \cdot 4) \mathrm{mmol})$, needle acupuncture $(3.5(1.6-14.7) \mathrm{mmol})$ or by transcutaneous electric nerve stimulation (6.0 (0-25-7) mmol) (Figure (B)).

Peak acid output was significantly reduced by electroacupuncture $(0 \cdot 8(0 \cdot 2-5 \cdot 1) \mathrm{mmol} / \mathrm{h}$; $\mathrm{p}<0.05)$ and transcutaneous nerve stimulation $(3.6(1 \cdot 2-12 \cdot 0) \mathrm{mmol} / \mathrm{h} ; \mathrm{p}<0.05)$. Peak acid output during the control period was $7 \cdot 6$ $(3 \cdot 4-12 \cdot 1) \mathrm{mmol} / \mathrm{h}$, during sham acupuncture $9 \cdot 1(5 \cdot 5-12 \cdot 9) \mathrm{mmol} / \mathrm{h}$, during laser acupuncture $5.5(0 \cdot 7-9 \cdot 0) \mathrm{mmol} / \mathrm{h}$, and during needle acupuncture $7 \cdot 3(2 \cdot 4-25 \cdot 3) \mathrm{mmol} / \mathrm{h}$ (Figure (C)).

The gastric acid secretion stimulated by sham feeding was significantly reduced by two forms of acupuncture - electroacupuncture and transcutaneous electric nerve stimulation. The inhibitory effect was more pronounced during the first 30 minute period, where median values of gastric acid secretion obtained during control period were reduced to $42 \cdot 1 \%$ by electroacupuncture and to $26 \cdot 3 \%$ by transcutaneous electric nerve stimulation. The effect of electroacupuncture lasted longer than transcutaneous nerve stimulation and was significant also during the second 30 minute period. Two further forms of acupuncture classic needle acupuncture and laser acupuncture - did not prove effective in the acute
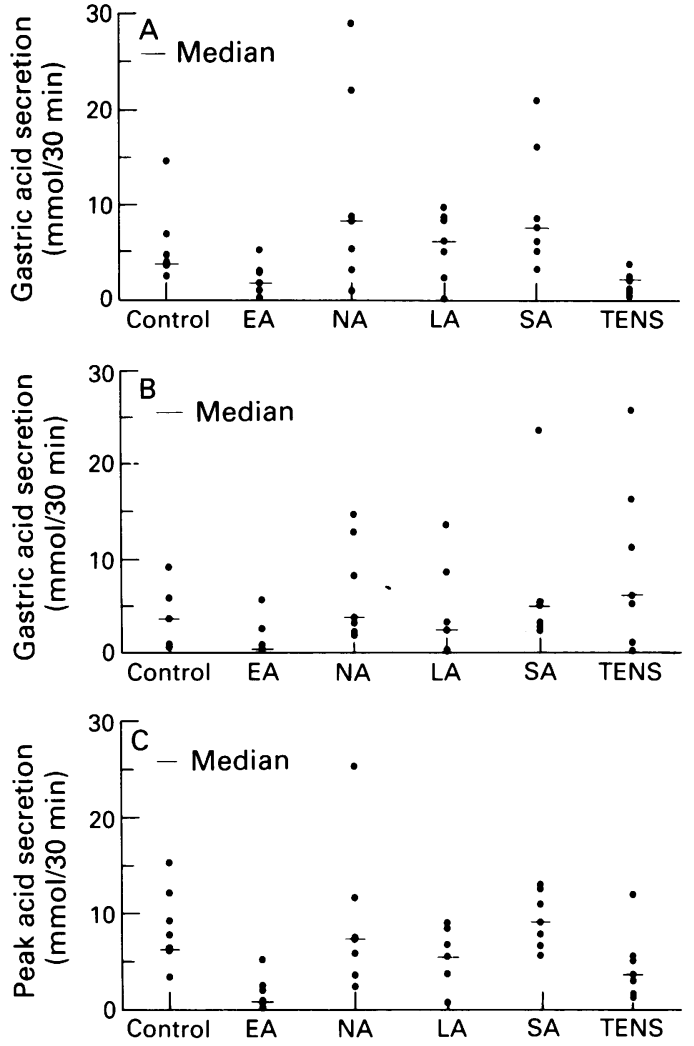

Gastric acid secretion during the first period $(A)$ 0-30 minutes after sham feeding and second $(B)$ period (30-60 minutes) and for peak acid secretion (C); values given as medians with their range for the control period (control), electroacupuncture (EA), needle acupuncture $(N A)$, laser acupuncture ( $L A)$, sham acupuncture $(S A)$, and transcutaneous electric nerve stimulation (TENS); $(A)$ : $E A$ and TENS significantly different $(p<0.05) \mathrm{v}$ control; (B) $E A$ significantly different $(p<0.05)$ v control; (C) $E A$ and TENS significantly different $(p<0.05) \mathrm{v}$ control.

experiment with healthy test subjects. Also, sham acupuncture entailing the insertion of needles at sites not defined as effective acupuncture points did not have any influence on gastric acid secretion stimulated by sham feeding.

\section{Discussion}

In this study, sham feeding stimulated gastric acid secretion was significantly reduced by electroacupuncture and transcutaneous electric nerve stimulation, the two forms of acupuncture that entail the most potent stimulation. Classic needle acupuncture, laser acupuncture, and sham acupuncture had no influence on gastric acid secretion. In previous studies effects were achieved by electroacupuncture $^{12}$ and classic needle acupuncture, ${ }^{10}$ and in animal experiments by electroacupuncture. ${ }^{13}$ The same acupuncture points were used as in this study. ${ }^{10121317}$

In this study the influence of electroacupuncture on gastric acid secretion is not explained merely by inserting the needles or by the electrostimulatory effect, as these procedures applied at other sites (sham acupuncture) led to no reduction in acid output. Although gastric acid secretion can be significantly inhibited, for example by hypnosis, ${ }^{18}$ in this study, 'suggestion' can be ruled out as an important factor, as in the 
remaining acupuncture procedures with more mild stimulation, no significant action on acid secretion was found.

Li et al ${ }^{12}$ stimulated gastric acid secretion by sham feeding in healthy subjects and reported that electroacupuncture reduced basal acid output significantly by 23.8 per cent and maximal acid output by 71 per cent.

Also in duodenal ulcer patients, classic needle acupuncture significantly reduced gastric acid secretion with a reduction rate of about $45-75 \% .{ }^{10}$ In earlier experiments, ${ }^{13}$ it was found that with classic needle acupuncture, and more noticeably with electroacupuncture, a significant influence on gastric acid secretion in the dog was obtained: in addition to the reduction in acid secretion, an increase in bicarbonate secretion was also found. Anti-muscarinic agents, but also local anaesthetics applied to the insertion points of the acupuncture needles abrogated this effect, so that the authors concluded that the effect of acupuncture was mediated by somatic afferent visceral fibres.

Acupuncture is considered to have some value in the treatment of ulcers. In four patients with duodenal ulcers resistant to medical treatment healing was seen after six to seven acupuncture sessions. ${ }^{11}$ Duodenal ulcer patients became complaint free after acupuncture. ${ }^{10}$ As the number of patients in both studies is limited, the effect of acupuncture on ulcer healing has yet to be proved.

The stimulation of gastric acid secretion by sham feeding entails mainly the central vagal component of gastric acid stimulation. ${ }^{19}$ Acupuncture seems to reduce gastric acid secretion by afferent visceral fibres. ${ }^{13}$ At present there can only be speculation about the mechanisms produced by acupuncture, as regulation of gastric acid secretion is complex including neural, hormonal, and paracrine mechanisms. ${ }^{20}$ It cannot be excluded that at least part of the effect of acupuncture on gastric secretion also entails stimulation of bicarbonate secretion. ${ }^{13}$

At present, the treatment available for reducing gastric acid secretion is based on pharmacological blocking of parietal cell receptors for histamine, muscarine, gastrin, and inhibition of $\mathrm{H}^{+} / \mathrm{K}^{+}$-ATPase. ${ }^{20}$ The possi- bility of inhibiting gastric acid secretion by electroacupuncture, as shown in this study, offers an alternative to drug related reduction of gastric acid secretion. Our results could give a rational basis for peptic ulcer treatment only if the effect of electroacupuncture would last for a period longer than two hours. Furthermore, the effect of electroacupuncture on ulcer healing has yet to be proved in controlled trials.

The authors express their gratitude for statistical help to PD Dr med Annelore Altendorf-Hofmann, Centre of Statistical Tumour Evaluation, University of Erlangen-Nuremberg.

1 Rubin P. Therapeutic acupuncture: a selective review. South Med f 1977; 70: 974-7.

Kampik G, Bertsche O. Akupunktur bei inneren Krankheiten. Internist 1988; 29: 479-86.

3 Cunxin Y, Renming L, Jun Z, Naishi J, Dazheng Z. The curative effect and mechanism of action of the acupoints pishu and weishu. Tradit Chin Med 1986; 6: 249-52.

4 Jobst K. Acupuncture, asthma and breathlessness. Lancet 1986; ii: 1427.

5 Institute for acute abdominal diseases, Nan Kai Hospital, Tiensin and Acute Abdominal Condition Research Group, Tsunyi Medical College, Kweicho. Treatment of acute abdominal diseases by combined traditional Chinese and western medicine. Chin Med f 1978; 4: 11-6.

6 Nan Kai Hospital, Tiensin. Treatment of acute perforation of peptic ulcer with combined traditional Chinese and of peptic ulcer with combined traditional Chines

7 Liu JX, Zhao Q. Effect of acupuncture on intestinal motion and sero-enzyme activity in perioperation. Chung Hsi I Chieh Ho Trsa Chih 1991; 11: 133-4.

8 Matsumoto T. Evaluation of acupuncture in management of gastrointestinal atony. Surg Forum 1972; 23: 401-2.

9 Matsumoto T, Hayes F. Acupuncture, electric phenomenon of the skin, and postvagotomy gastrointestinal atony. Am $\mathcal{F}$ Surg 1973; 125: 176-80.

10 Sodipo J Falaiye JM. Acupuncture and gastric acid studies. Am f Chin Med 1979; 7: 356-61.

11 Salvi E, Pistilli A, Romiti P, Bedogni G, Pedrazzoli C. Ulcera duodenale. Aspetti gastroscopici prima e dopo trattamento agopunturale. Minerva Med 1984; 74: trattamento

$12 \mathrm{Li} \mathrm{YY,} \mathrm{Cherverton} \mathrm{SG,} \mathrm{Tougas} \mathrm{G,} \mathrm{Hunt} \mathrm{RH.} \mathrm{Acupuncture}$ inhibits the response to sham feeding. Gut 1988; 29: A724.

13 Zhou L, Chey WY. Electric acupuncture stimulates nonparietal cell secretion of the stomach in dog. Life Sci 1984; 34: 2233-8.

14 Knutson U, Olbe L. Gastric acid response to sham feeding in the duodenal ulcer patient. Scand $\mathcal{f}$ Gastroenterol 1973; 8: 513-22.

15 Essentials of Chinese Acupuncture. Beijing: Academy of Traditional Chinese Medicine, 1980.

16 Baron JH. Studies of basal and peak acid output with an augmented histamine test. Gut 1963; 4: 136-44.

17 Gideon L. Acupuncture: clinical trials in the horse. $f \mathrm{Am}$ Vet Med Assoc 1977; 170: 220-4.

$18 \mathrm{Klein} \mathrm{KB}$, Spiegel D. Modulation of gastric acid secretion by hypnosis. Gastroenterology 1989; 96: 1383-7.

19 Feldman M, Richardson CT. Role of thought, sight, smell and taste of food in the cephalic phase of gastric acid secretion in humans. Gastroenterology 1986; 90: 428-33.

20 Schubert ML, Shamburek RD. Control of acid secretion. In: Hunt RH, ed. Peptic ulcer disease. Gastroenterol Clin North Am 1990; 19: 1-25. 